\title{
RAS Family Oncogene
}

National Cancer Institute

\section{Source}

National Cancer Institute. RAS Family Oncogene. NCI Thesaurus. Code C17061.

A family of oncogenes that are highly homologous with the retrovirus-associated DNA sequences (ras) originally isolated from Harvey and Kirsten murine sarcoma viruses.

Mutated or overexpressed forms of the human ras genes are associated with constitutive ras GT Pase activity, increased cellular proliferation, and cell transformation. 